\title{
Mutual Influence of Pnicogen Bonds and Beryllium Bonds: Energies and Structures in the Spotlight
}

Ibon Alkorta, ${ }^{\mathrm{a}, *}$ José Elguero, ${ }^{\mathrm{a}}$ Janet E. Del Bene, ${ }^{\mathrm{b}, *}$ Otilia Mó, ${ }^{\mathrm{c}}$ M. Merced Montero-Campillo, ${ }^{\mathrm{c}} *$ Manuel Yáñez

a Instituto de Química Médica, CSIC, Juan de la Cierva, 3, E-28006 Madrid, Spain, ibon@iqm.csic.es

${ }^{\mathrm{b}}$ Department of Chemistry, Youngstown State University, Youngstown, Ohio 44555 USA, jedelbene@ysu.edu

c Departamento de Química, Facultad de Ciencias, Módulo 13, and Institute of Advanced Chemical Sciences (IadChem). Universidad Autónoma de Madrid, Campus de Excelencia UAM-CSIC, Cantoblanco, E-28049 Madrid, Spain, mm.montero@uam.es

Index:

Pg. S2 Table S1. Binary N-base: PH2F complexes

Pg. S3-S4 Table S2. Binary PH2F:BeX2 (X= H, F, Cl) complexes

Pg. S5-S7 Table S3. Ternary H3N:PH2F:BeX2 (X= H, F, Cl) complexes

Pg. S8-S10 Table S4. Ternary NHCH2:PH2F:BeX2 (X= H, F, Cl) complexes

Pg. S11-S13 Table S5. Ternary HCN:PH2F:BeX2 (X= H, F, Cl) complexes

Pg. S14 Table S6. Binary PH2F:BeX2 (BeX2 = BeCO3, BeSO4) complexes

Pg. S15-S16 Table S7. Ternary N-base:PH2F:BeX2 (BeX2 = BeCO3, BeSO4) complexes

Pg. S17 Table S8. Ab initio dissociation energies, predicted values with Eq. 3 and residues $\left(\mathrm{kJ} \mathrm{mol}^{-1}\right)$.

Pg. S18 Fig. S1. F ‥Be vs. P...N distances $(\AA \AA)$ in the ternary complexes.

Pg. S19 Fig. S2. $\rho_{\text {ВСР }}(\mathrm{au})$ at the Be $\cdots$ F and P-F bonds vs. the interatomic distances, $\AA$. The exponential relationships are shown.

Pg. S20-S21 Table S9. Charge (e) of the monomers within the binary and ternary complexes. 
Table S1. Binary N-base: PH2F complexes

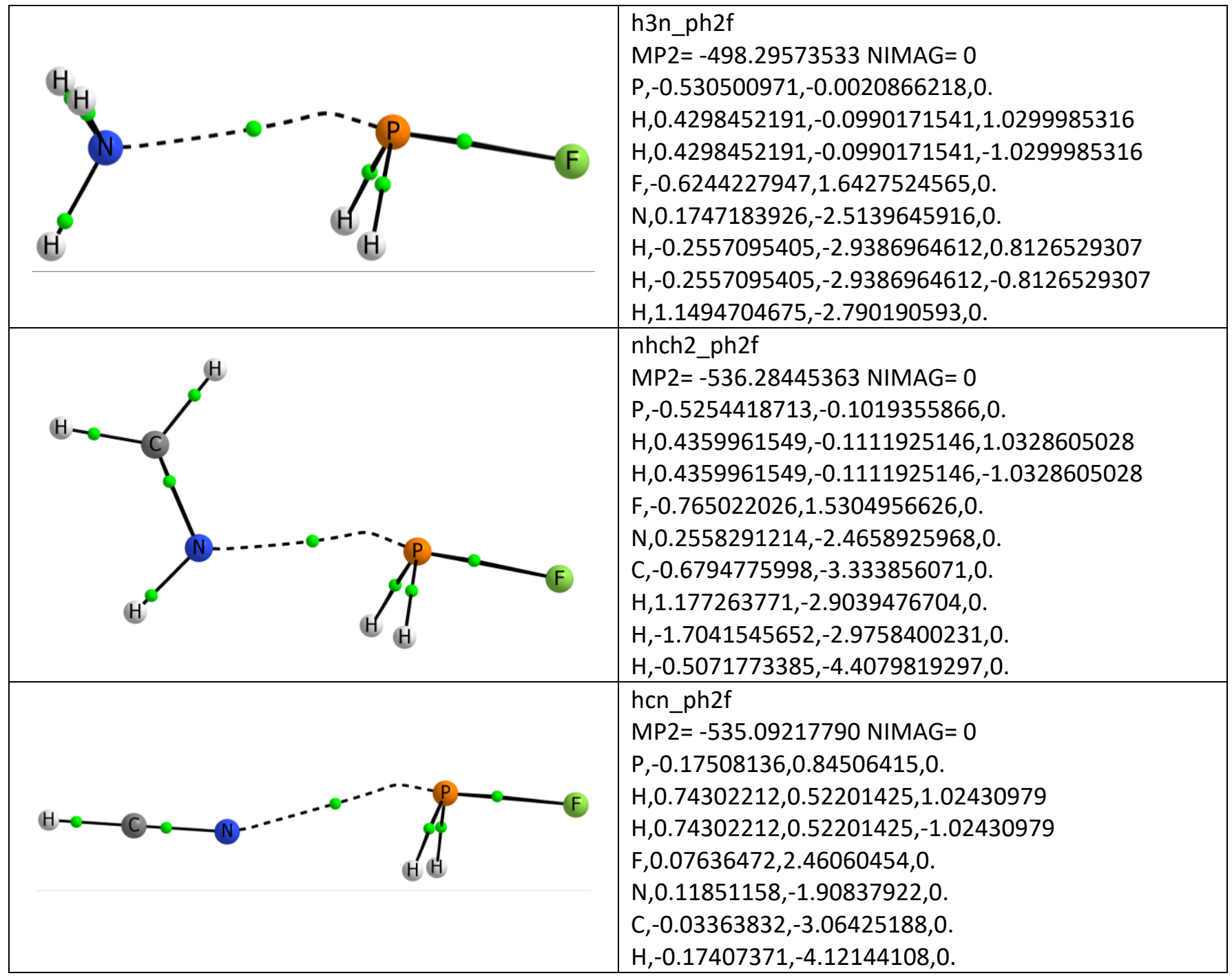


Table S2. Binary PH2F:BeX2 (X= H, F, Cl) complexes

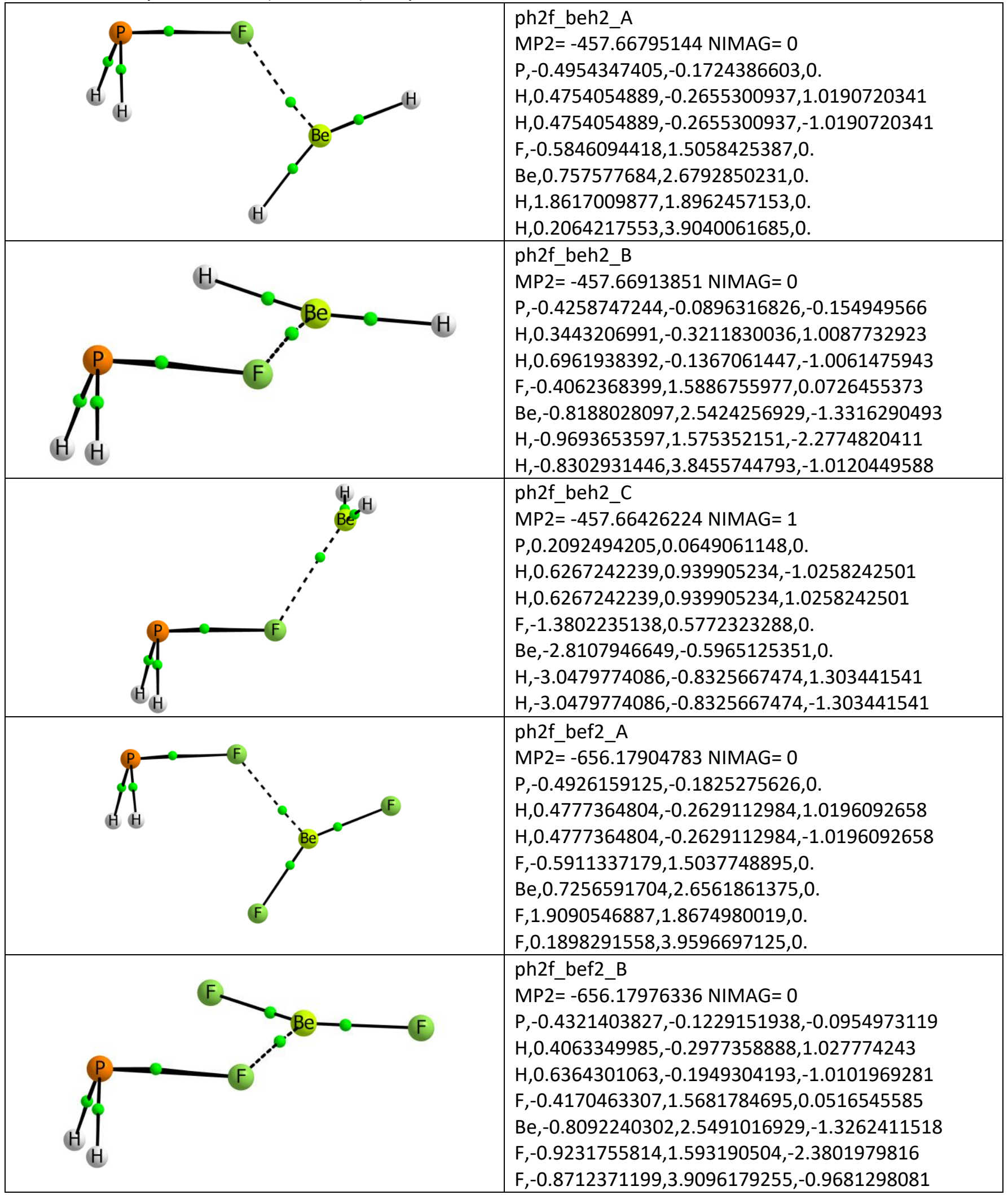




\begin{tabular}{|c|c|}
\hline $\mathrm{H}_{\mathrm{H}}$ & $\begin{array}{l}\text { ph2f_bef2_C } \\
\text { MP2= -656.17533571 NIMAG }=1 \\
P, 0.2381510527,0.0649912085,0 . \\
H, 0.6241326506,0.9530496329,-1.0260138538 \\
H, 0.6241326506,0.9530496329,1.0260138538 \\
F,-1.3721191925,0.5356989677,0 . \\
\text { Be,-2.7860495545,-0.5686417397,0. } \\
F,-3.0763944569,-0.8388940228,1.3557593709 \\
F,-3.0763944569,-0.8388940228,-1.3557593709\end{array}$ \\
\hline & $\begin{array}{l}\text { Ph2f_becl2_A } \\
\text { MP2= -1376.10637744 NIMAG }=0 \\
P,-0.5545403943,-0.2307582354,0 . \\
H, 0.40998485,-0.3649006756,1.0196302343 \\
H, 0.40998485,-0.3649006756,-1.0196302343 \\
F,-0.5440839851,1.4709965511,0 . \\
\text { Be,0.6831242722,2.642481399,0. } \\
C l, 2.341658589,1.7927359889,0 . \\
C l,-0.0495659885,4.3374964318,0 .\end{array}$ \\
\hline $\mathrm{H}_{\mathrm{H}}$ & $\begin{array}{l}\text { ph2f_becl2_B } \\
\text { MP2= -1376.10741949 NIMAG=0 } \\
\text { P,-0.3916864102,-0.1952035127,-0.0620495059 } \\
\text { H,0.3983174064,-0.2788909886,1.1064345654 } \\
\text { H,0.7197741778,-0.296969359,-0.9217694158 } \\
\text { F,-0.3866541978,1.5168549091,-0.0005786909 } \\
\text { Be,-0.8140050253,2.5219044717,-1.2759284705 } \\
\text { Cl,-1.0782896726,1.4409508295,-2.7785481141 } \\
\text { Cl,-0.8575146183,4.29586074,-0.7683947482 }\end{array}$ \\
\hline$\left.\mathbb{H}\right|_{H}$ & $\begin{array}{l}\text { ph2f_becl2_C } \\
\text { MP2= -1376.10318563 NIMAG }=1 \\
\text { P,0.2406766595,0.0659666918,0. } \\
H, 0.638587068,0.9468696711,-1.0270823868 \\
H, 0.638587068,0.9468696711,1.0270823868 \\
\text { F,-1.3695465731,0.5975517876,0. } \\
\text { Be,-2.7066113112,-0.4795978096,0. } \\
\text { Cl,-3.1341615969,-0.9079097108,1.751383047 } \\
\text { Cl,-3.1341615969,-0.9079097108,-1.751383047 }\end{array}$ \\
\hline
\end{tabular}


Table S3. Ternary H3N:PH2F:BeX2 (X= H, F, Cl) complexes

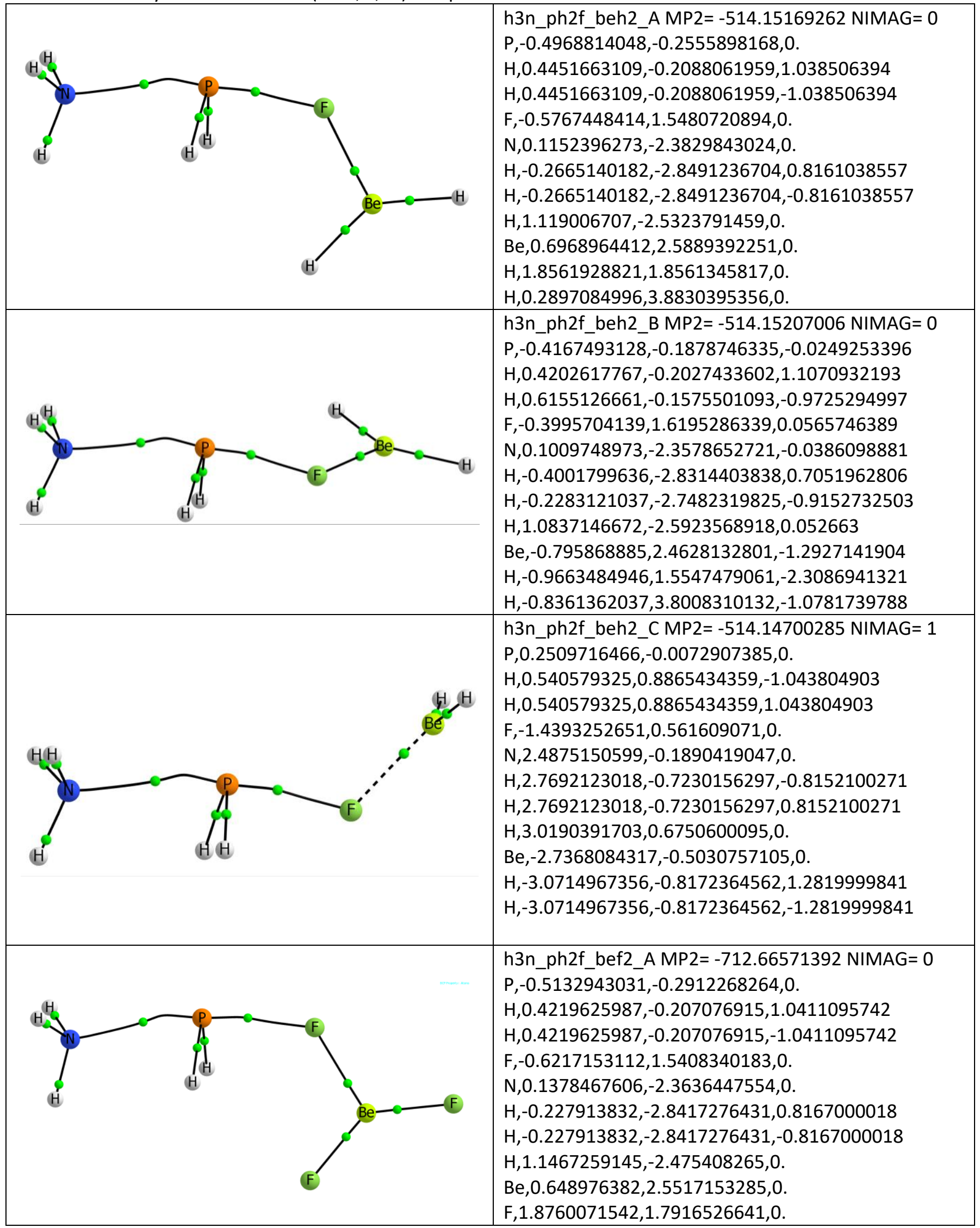




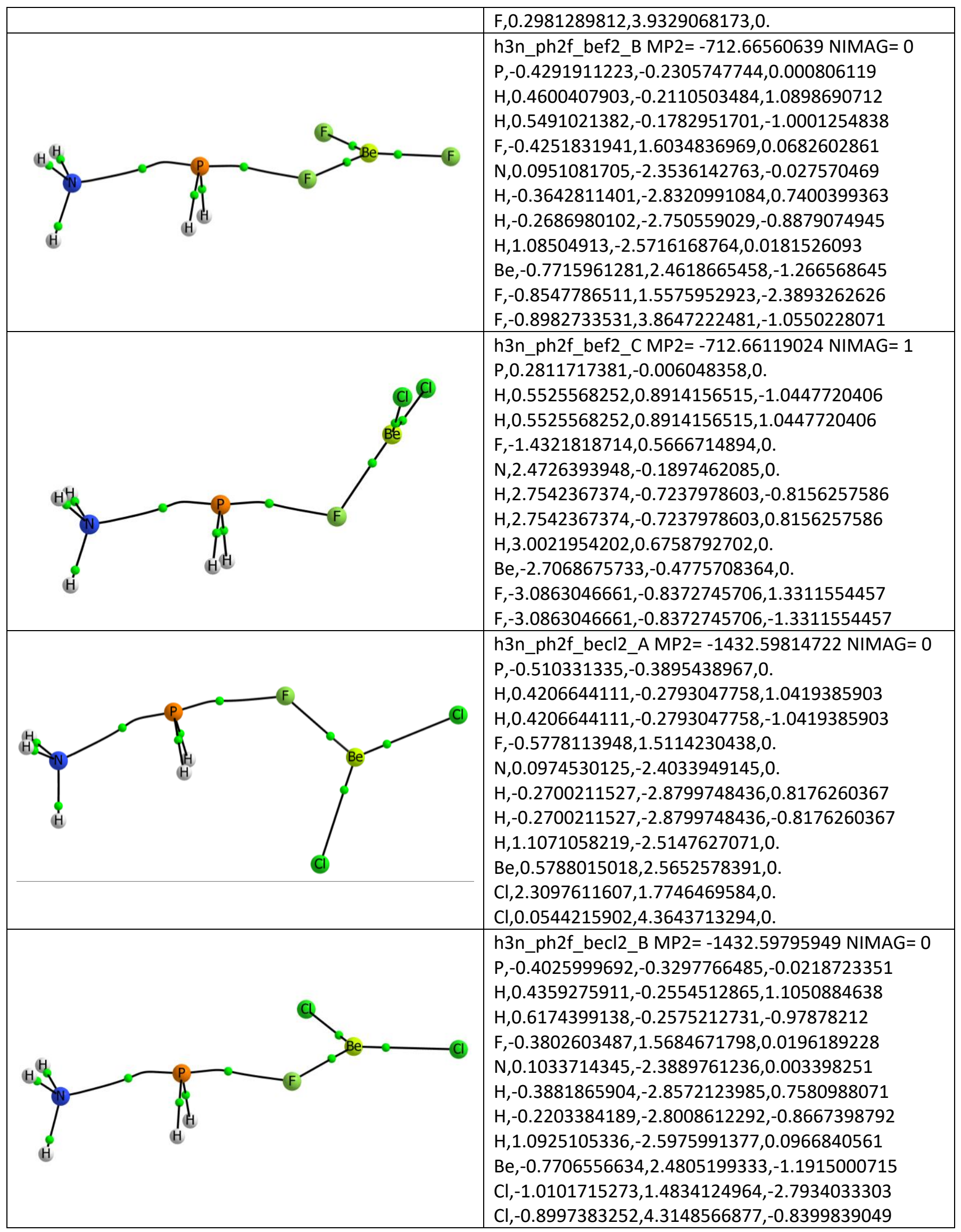




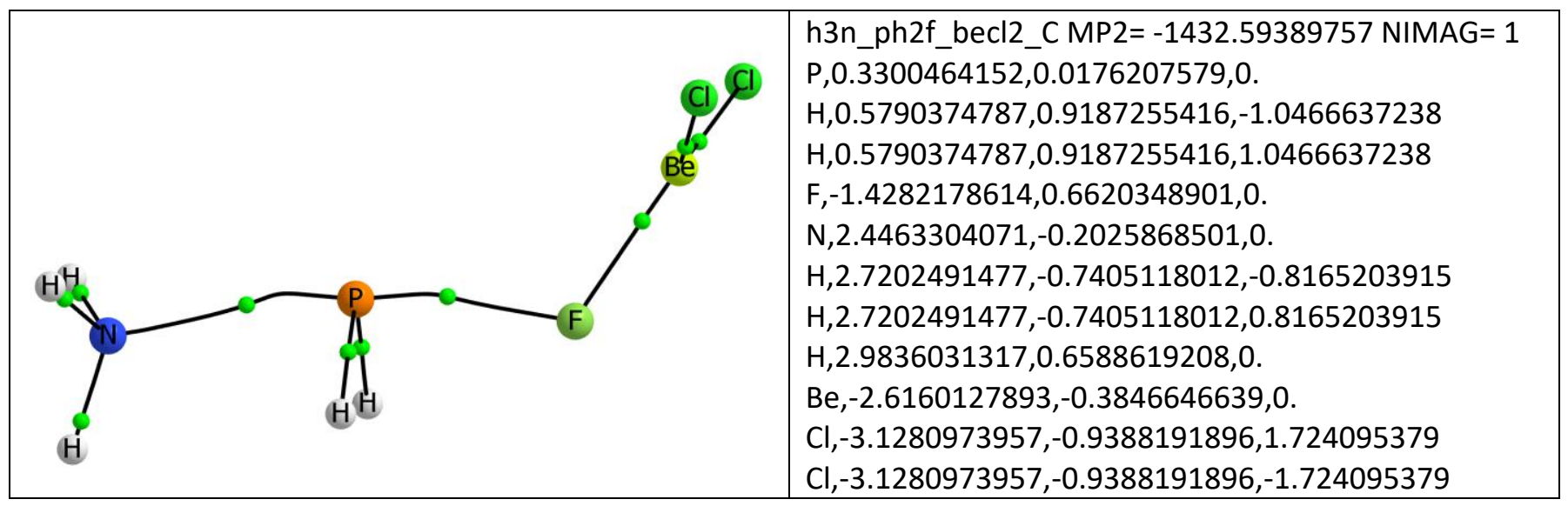


Table S4. Ternary NHCH2:PH2F:BeX2 (X= H, F, Cl) complexes

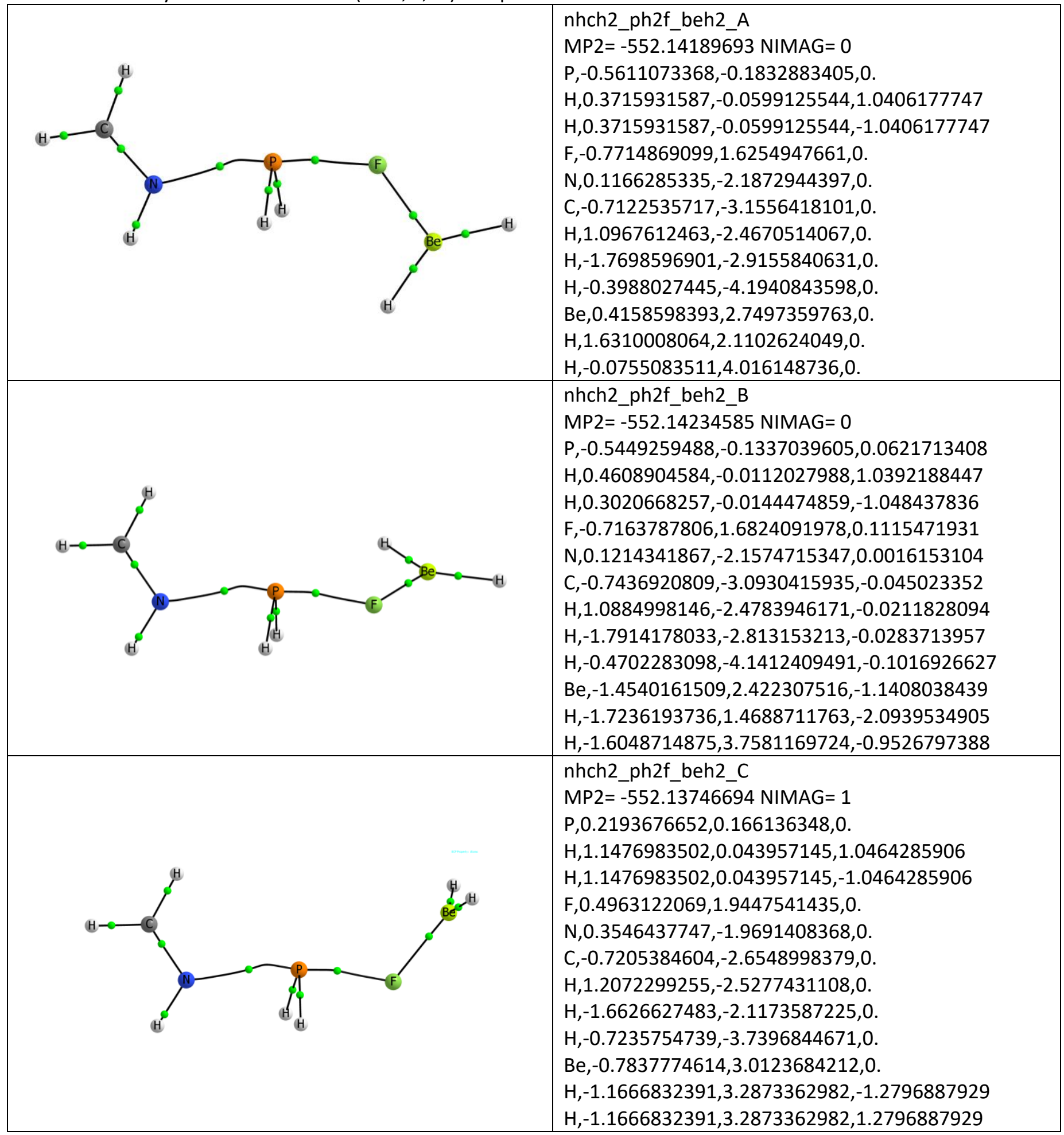




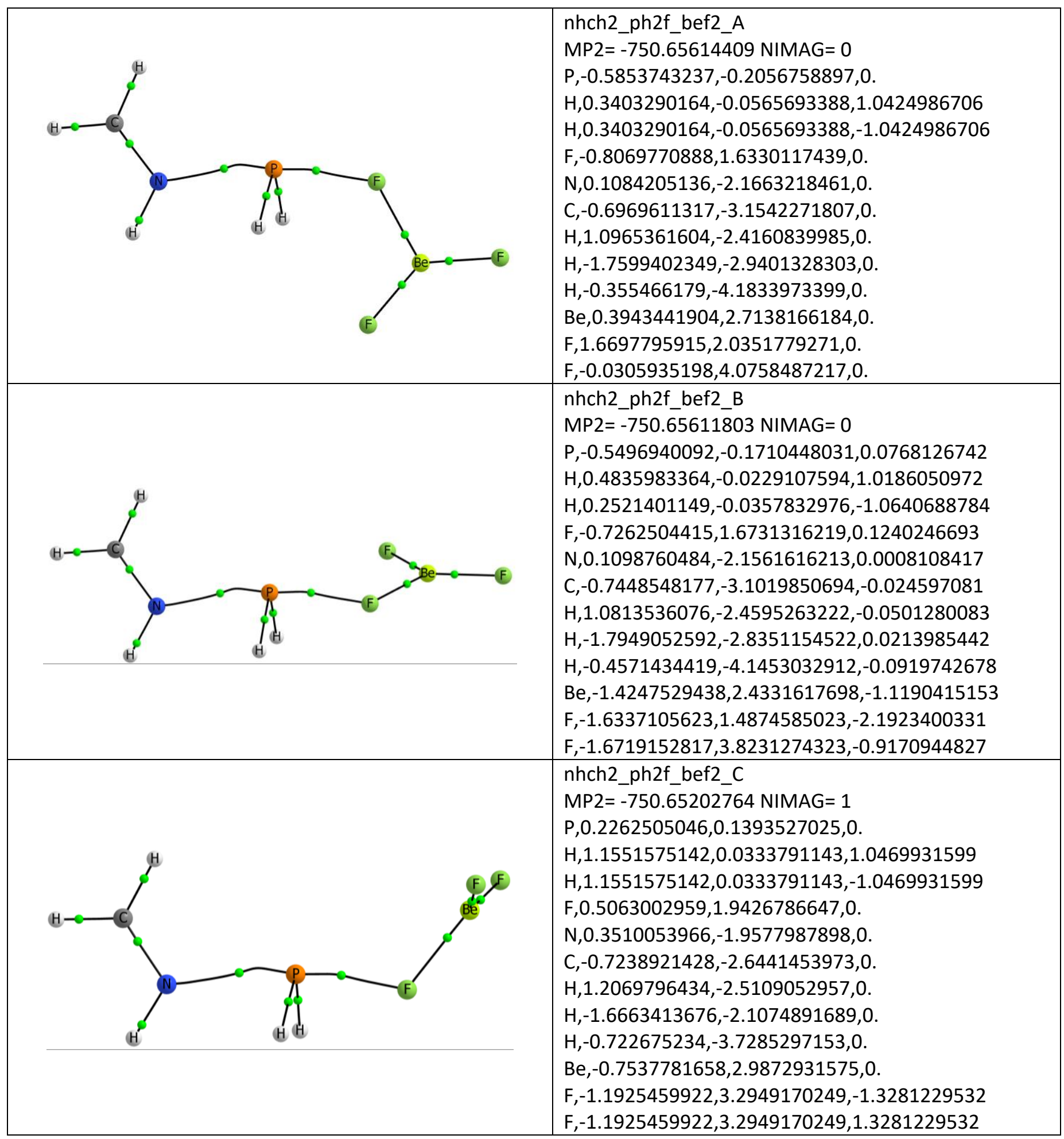




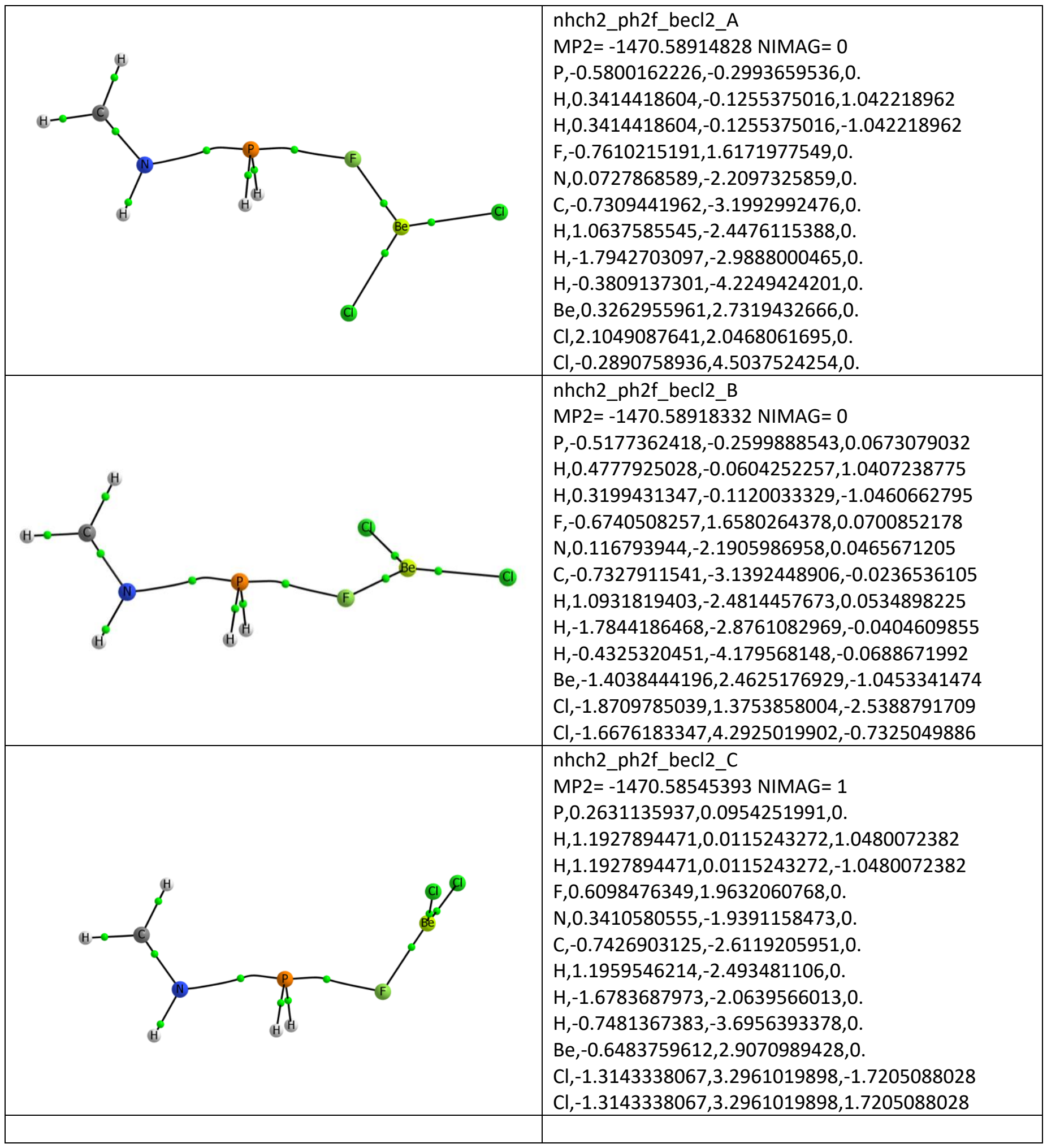


Table S5. Ternary HCN:PH2F:BeX2 (X= H, F, Cl) complexes

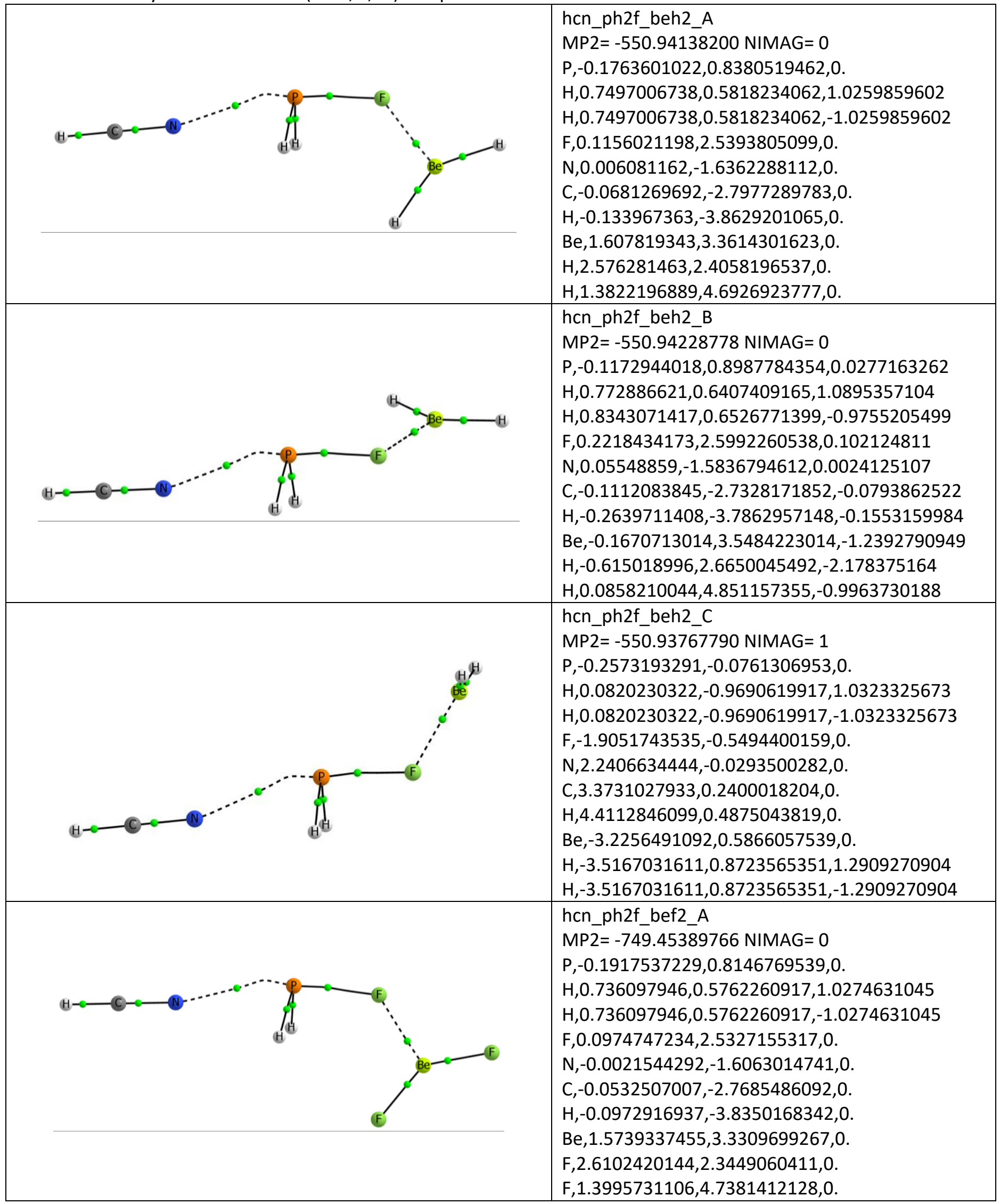




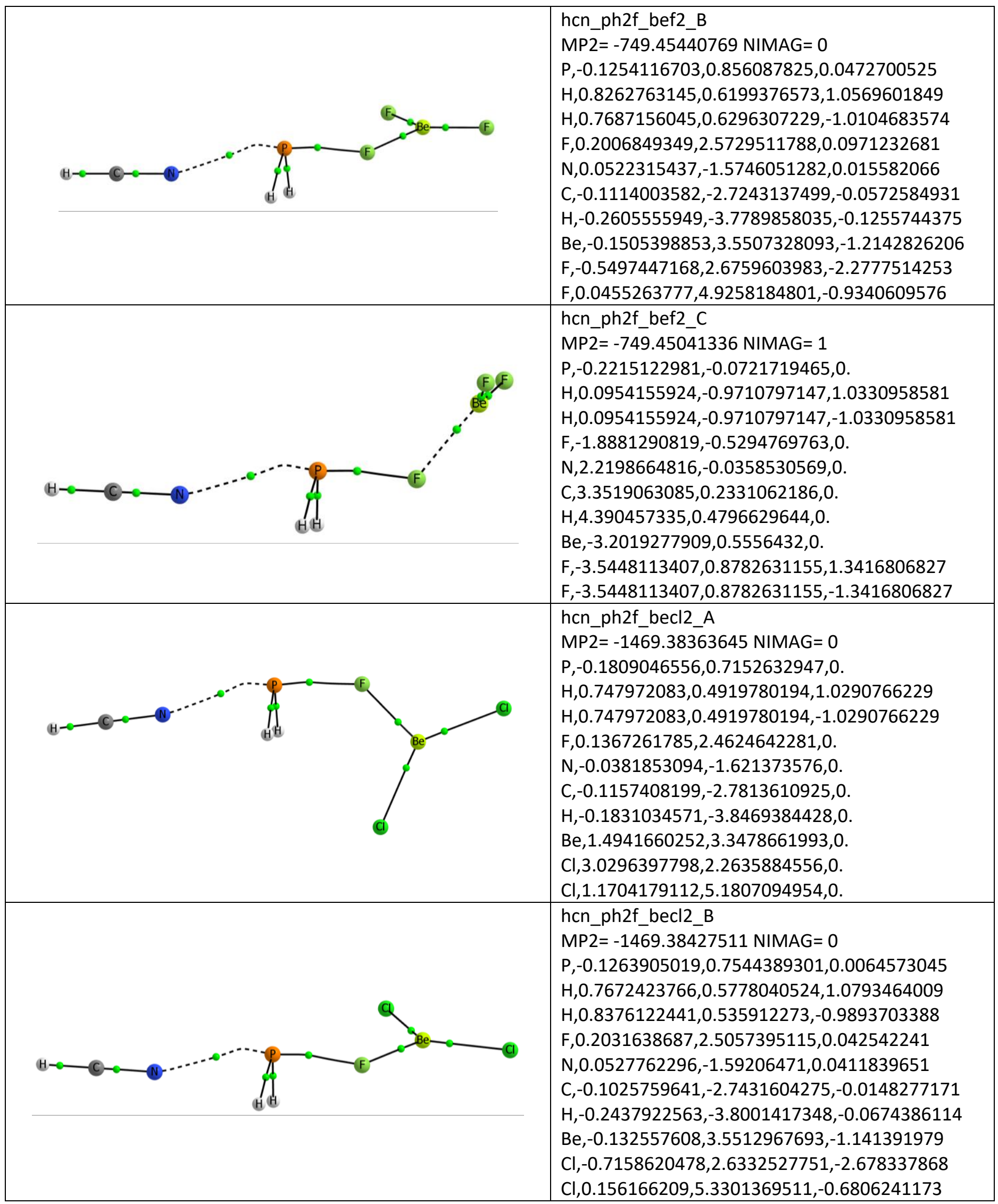




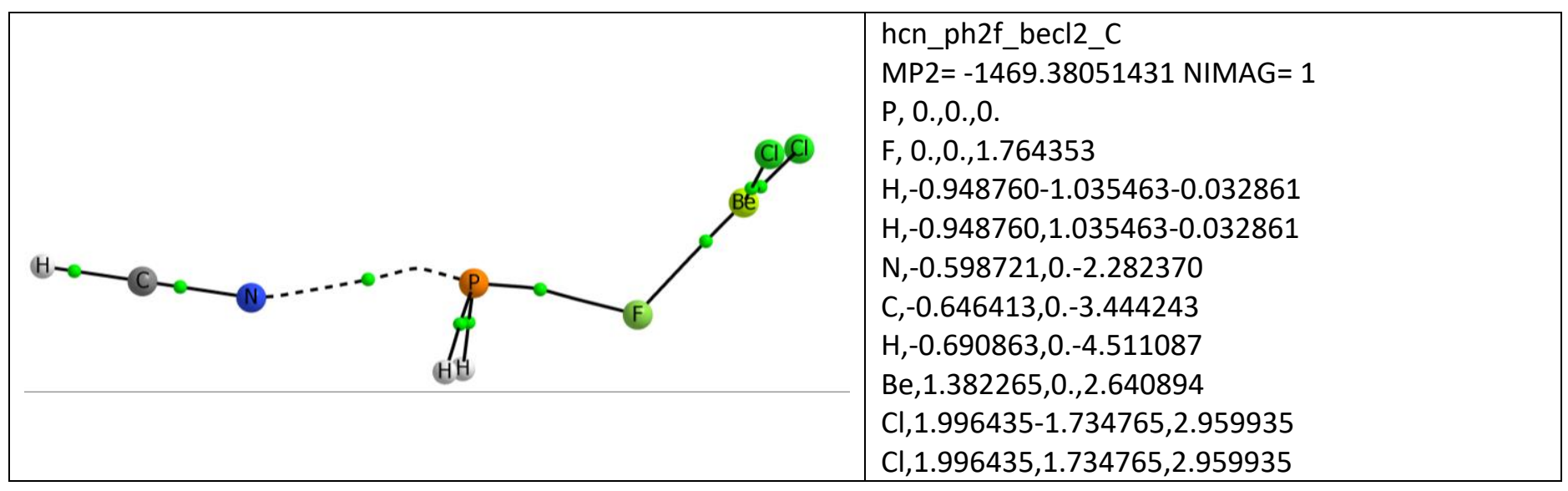


Table S6. Binary PH2F:BeX2 (BeX2 = BeCO3, BeSO4) complexes

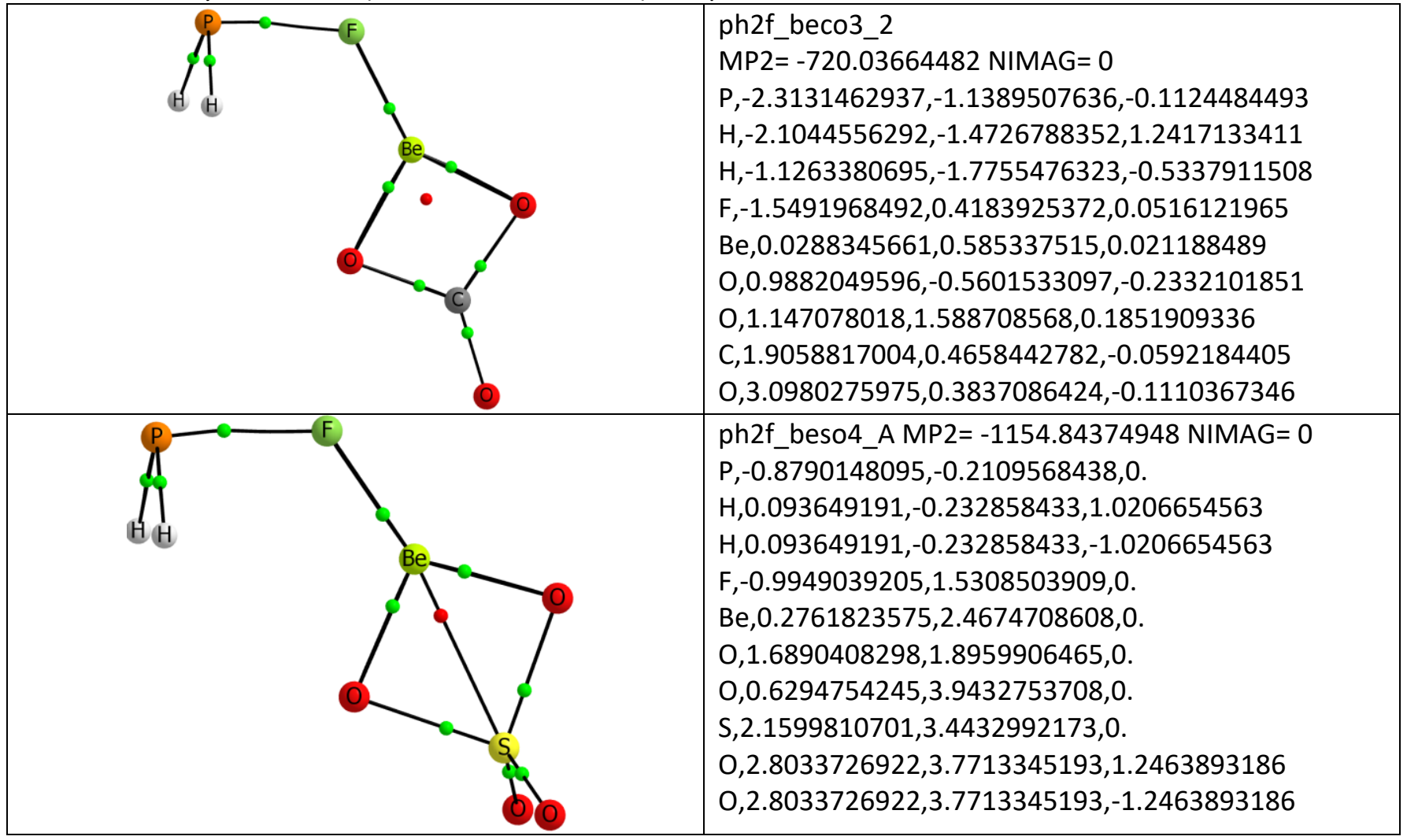


Table S7. Ternary N-base:PH2F:BeX2 (BeX2 = BeCO3, BeSO4) complexes

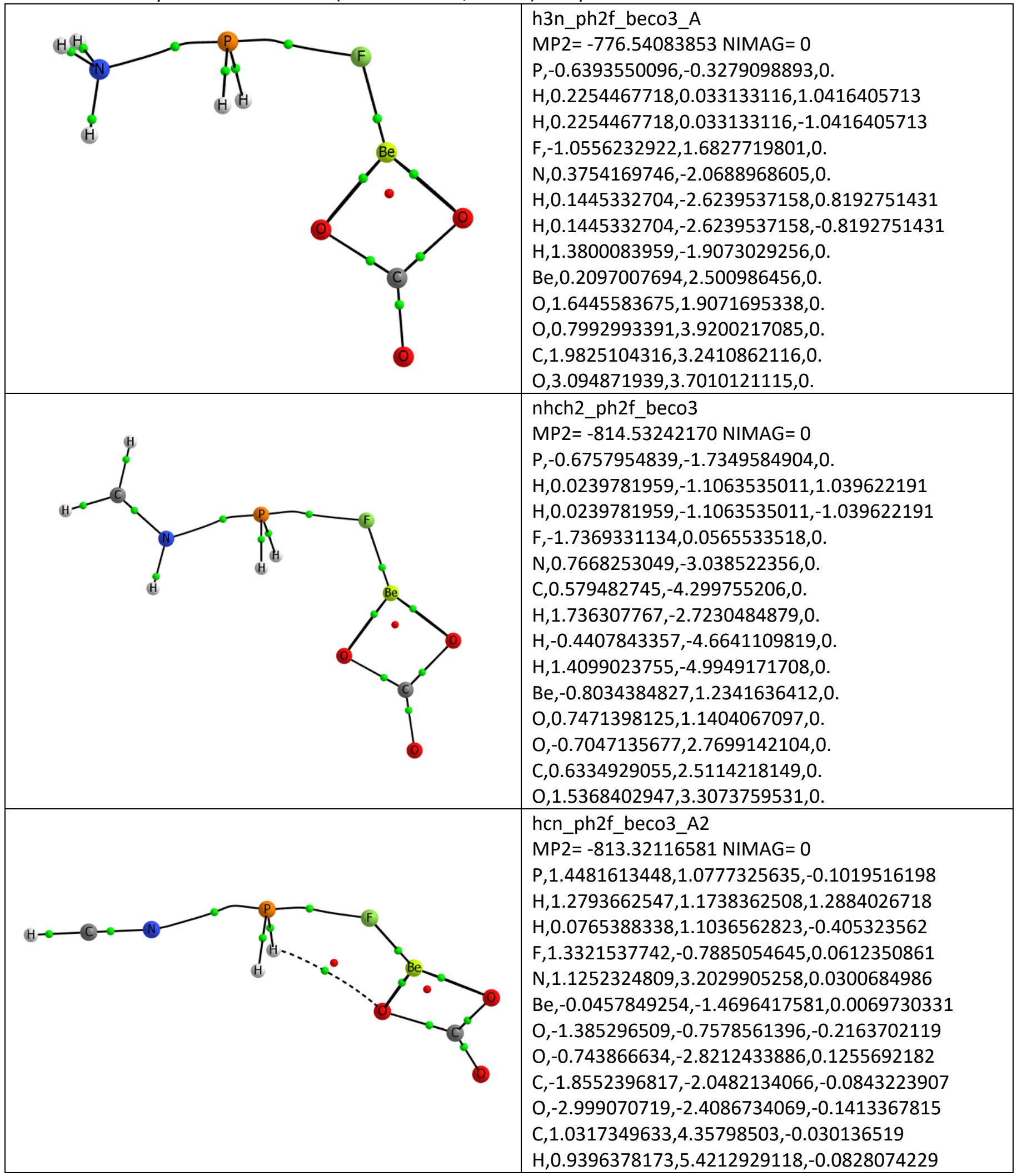




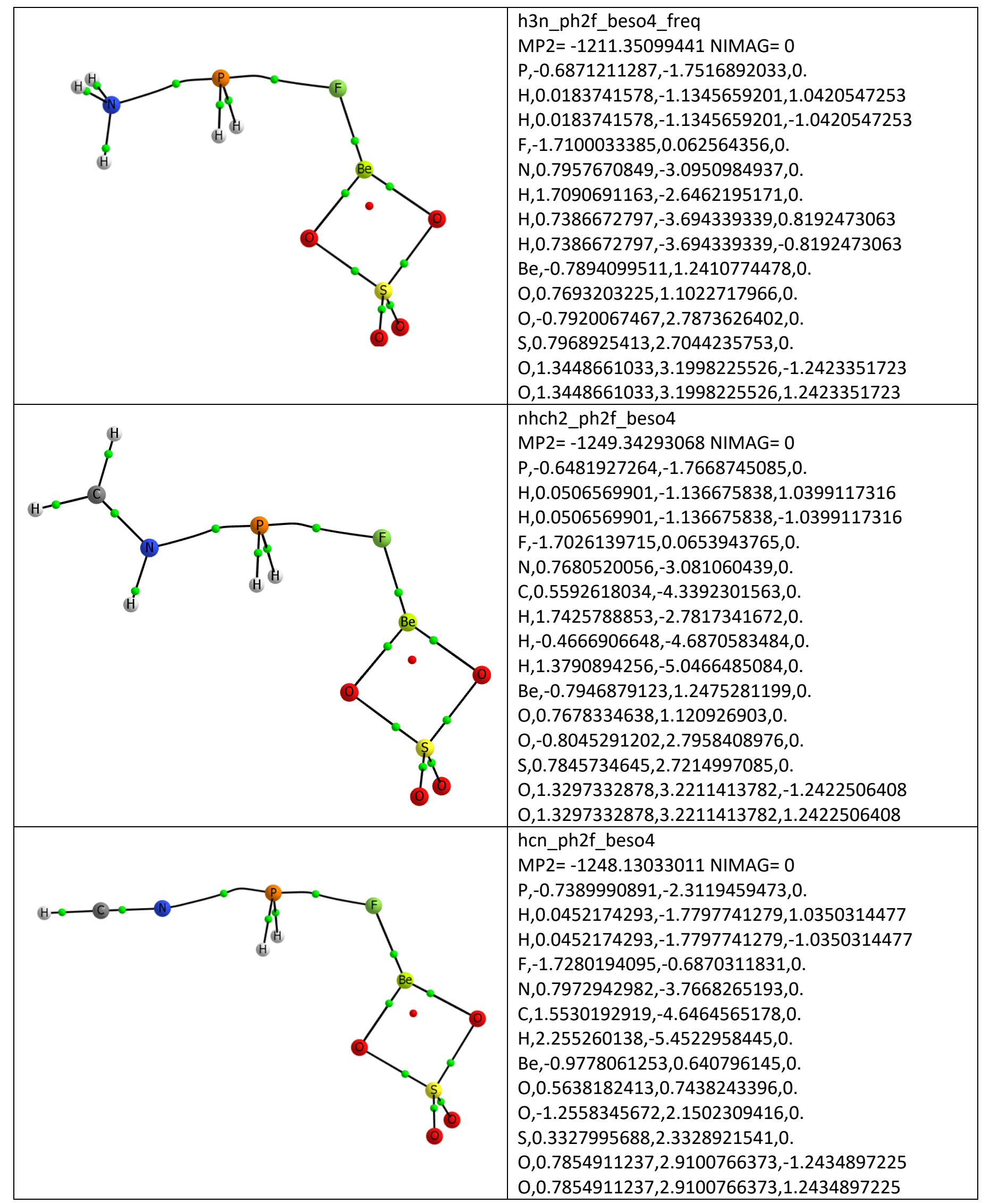


Table S8. Ab initio dissociation energies, predicted values with Eq. 3 and residues $\left(\mathrm{kJ} \mathrm{mol}^{-1}\right)$.

\begin{tabular}{|l|c|c|c|}
\hline & De & Predicted & Residues \\
\hline $\mathrm{H}_{3} \mathrm{~N}: \mathrm{PH}_{2} \mathrm{~F}: \mathrm{BeH}_{2}(\mathrm{~A})$ & 91.6 & 92.3 & -0.7 \\
\hline $\mathrm{H}_{3} \mathrm{~N}: \mathrm{PH}_{2} \mathrm{~F}: \mathrm{BeH}_{2}(\mathrm{~B})$ & 92.6 & 93.1 & -0.5 \\
\hline $\mathrm{H}_{3} \mathrm{~N}: \mathrm{PH}_{2} \mathrm{~F}: \mathrm{BeH}_{2}(\mathrm{C})$ & 79.3 & 81.8 & -2.5 \\
\hline $\mathrm{H}_{3} \mathrm{~N}: \mathrm{PH}_{2} \mathrm{~F}: \mathrm{BeF}_{2}(\mathrm{~A})$ & 113.1 & 112.5 & 0.6 \\
\hline $\mathrm{H}_{3} \mathrm{~N}: \mathrm{PH}_{2} \mathrm{~F}: \mathrm{BeF}_{2}(\mathrm{~B})$ & 112.8 & 113.3 & -0.5 \\
\hline $\mathrm{H}_{3} \mathrm{~N}: \mathrm{PH}_{2} \mathrm{~F}: \mathrm{BeF}_{2}(\mathrm{C})$ & 101.2 & 102.0 & -0.8 \\
\hline $\mathrm{H}_{3} \mathrm{~N}: \mathrm{PH}_{2} \mathrm{~F}: \mathrm{BeCl}_{2}(\mathrm{~A})$ & 129.5 & 127.4 & 2.1 \\
\hline $\mathrm{H}_{3} \mathrm{~N}: \mathrm{PH}_{2} \mathrm{~F}: \mathrm{BeCl}_{2}(\mathrm{~B})$ & 129.0 & 128.2 & 0.8 \\
\hline $\mathrm{H}_{3} \mathrm{~N}: \mathrm{PH}_{2} \mathrm{~F}: \mathrm{BeCl}_{2}(\mathrm{C})$ & 118.4 & 116.9 & 1.5 \\
\hline $\mathrm{CH}{ }_{2} \mathrm{HN}: \mathrm{PH}_{2} \mathrm{~F}: \mathrm{BeH}_{2}(\mathrm{~A})$ & 101.7 & 103.7 & -2.0 \\
\hline $\mathrm{CH}_{2} \mathrm{HN}: \mathrm{PH}_{2} \mathrm{~F}: \mathrm{BeH}_{2}(\mathrm{~B})$ & 102.8 & 104.5 & -1.7 \\
\hline $\mathrm{CH}{ }_{2} \mathrm{HN}: \mathrm{PH}_{2} \mathrm{~F}: \mathrm{BeH}_{2}(\mathrm{C})$ & 90.0 & 93.2 & -3.2 \\
\hline $\mathrm{CH}{ }_{2} \mathrm{HN}: \mathrm{PH}_{2} \mathrm{~F}: \mathrm{BeF}_{2}(\mathrm{~A})$ & 123.7 & 123.9 & -0.2 \\
\hline $\mathrm{CH}_{2} \mathrm{HN}: \mathrm{PH}_{2} \mathrm{~F}: \mathrm{BeF}_{2}(\mathrm{~B})$ & 123.7 & 124.7 & -1.0 \\
\hline $\mathrm{CH}{ }_{2} \mathrm{HN}: \mathrm{PH}_{2} \mathrm{~F}: \mathrm{BeF}_{2}(\mathrm{C})$ & 112.9 & 113.4 & -0.5 \\
\hline $\mathrm{CH}{ }_{2} \mathrm{HN}: \mathrm{PH}_{2} \mathrm{~F}: \mathrm{BeCl}_{2}(\mathrm{~A})$ & 141.7 & 138.8 & 2.9 \\
\hline $\mathrm{CH}{ }_{2} \mathrm{HN}: \mathrm{PH}_{2} \mathrm{~F}: \mathrm{BeCl}_{2}(\mathrm{~B})$ & 141.8 & 139.6 & 2.2 \\
\hline $\mathrm{CH}{ }_{2} \mathrm{HN}: \mathrm{PH}_{2} \mathrm{~F}: \mathrm{BeCl}_{2}(\mathrm{C})$ & 132.0 & 128.3 & 3.7 \\
\hline $\mathrm{HCN}: \mathrm{PH}_{2} \mathrm{~F}: \mathrm{BeH}_{2}(\mathrm{~A})$ & 64.9 & 62.1 & 2.8 \\
\hline $\mathrm{HCN}: \mathrm{PH}_{2} \mathrm{~F}: \mathrm{BeH}_{2}(\mathrm{~B})$ & 67.3 & 62.9 & 4.4 \\
\hline $\mathrm{HCN}: \mathrm{PH}_{2} \mathrm{~F}: \mathrm{BeH}_{2}(\mathrm{C})$ & 55.2 & 51.6 & 3.6 \\
\hline $\mathrm{HCN}: \mathrm{PH}_{2} \mathrm{~F}: \mathrm{BeF}_{2}(\mathrm{~A})$ & 82.4 & 82.3 & 0.1 \\
\hline $\mathrm{HCN}: \mathrm{PH}_{2} \mathrm{~F}: \mathrm{BeF}_{2}(\mathrm{~B})$ & 83.8 & 83.1 & 0.7 \\
\hline $\mathrm{HCN}: \mathrm{PH}_{2} \mathrm{~F}: \mathrm{BeF}_{2}(\mathrm{C})$ & 73.3 & 71.8 & 1.5 \\
\hline $\mathrm{HCN}: \mathrm{PH}_{2} \mathrm{~F}: \mathrm{BeCl}_{2}(\mathrm{~A})$ & 91.8 & 97.2 & -5.4 \\
\hline $\mathrm{HCN}: \mathrm{PH}_{2} \mathrm{~F}: \mathrm{BeCl}_{2}(\mathrm{~B})$ & 93.5 & 98.0 & -4.5 \\
\hline $\mathrm{HCN}: \mathrm{PH}_{2} \mathrm{~F}: \mathrm{BeCl}_{2}(\mathrm{C})$ & 83.6 & 86.7 & -3.1 \\
\hline
\end{tabular}




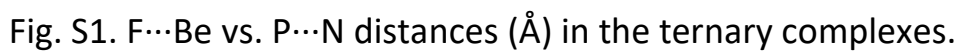

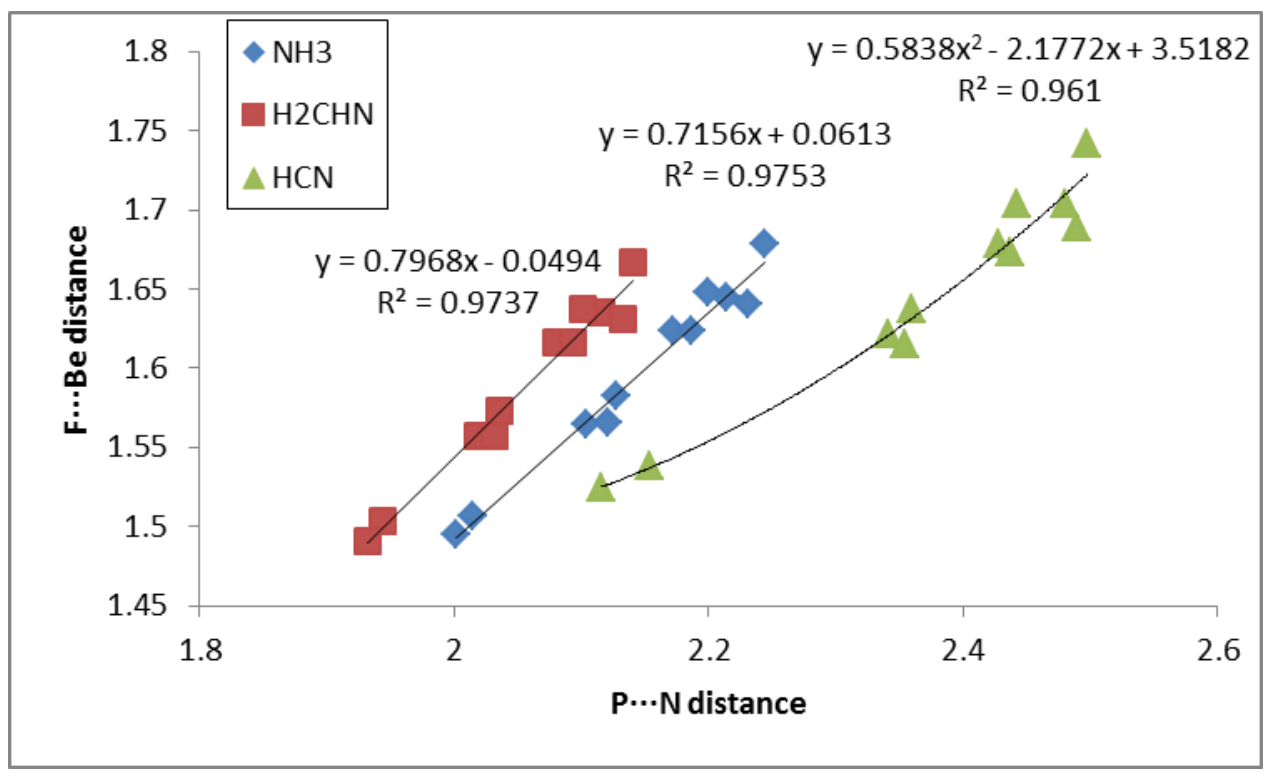


Fig. S2. $\rho_{\mathrm{BCP}}(\mathrm{au})$ at the Be $\cdots \mathrm{F}$ and P-F bonds vs. the interatomic distances, $\AA$. The exponential relationships are shown.
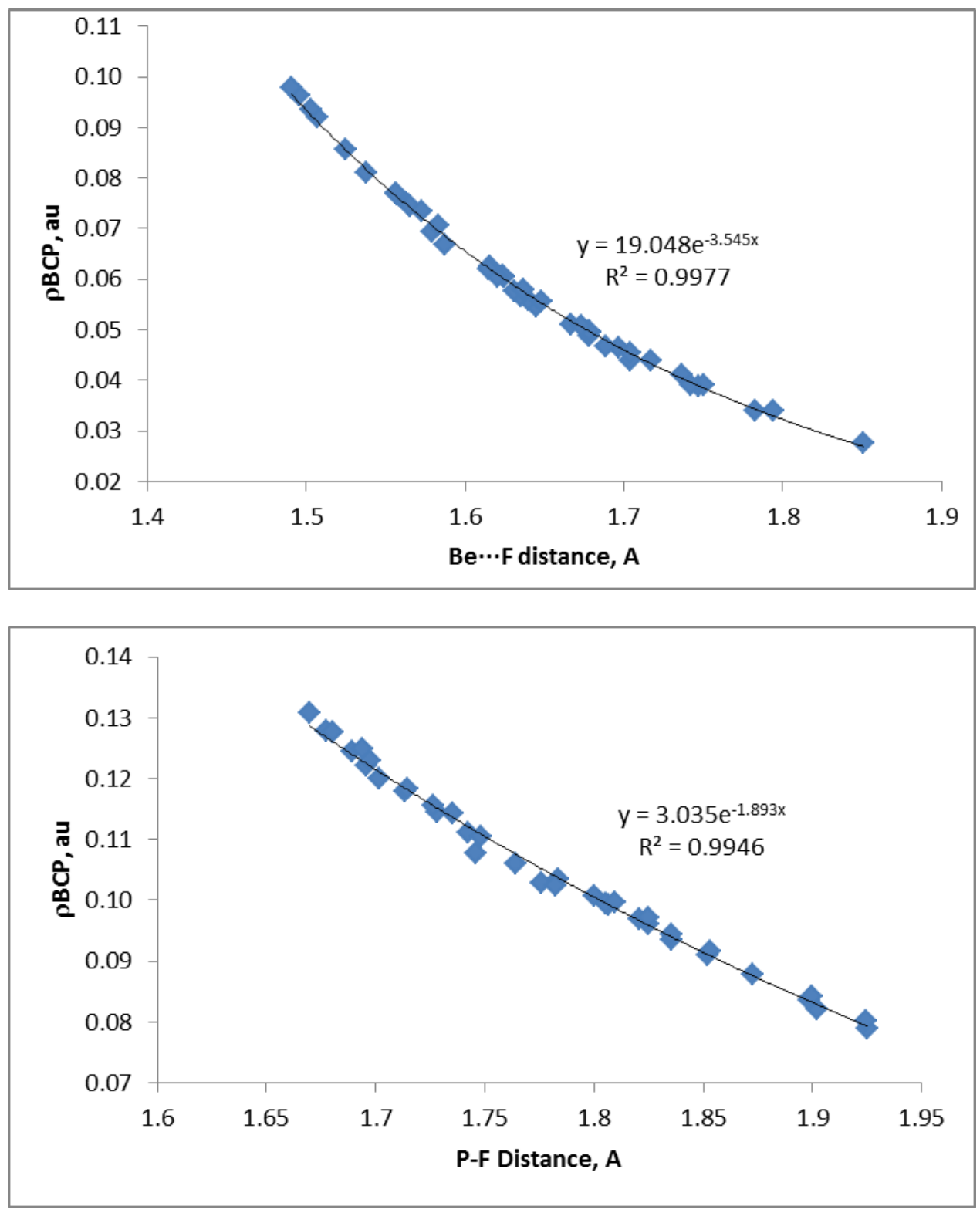
Table S9. Charge (e) of the monomers within the binary and ternary complexes

\begin{tabular}{|c|c|c|c|}
\hline & N-Base & $\mathrm{PH}_{2} \mathrm{~F}$ & $\mathrm{BeR}_{2}$ \\
\hline $\mathrm{H}_{3} \mathrm{~N}: \mathrm{PH}_{2} \mathrm{~F}$ & 0.072 & -0.072 & \\
\hline $\mathrm{CH}_{2} \mathrm{HN}: \mathrm{PH}_{2} \mathrm{~F}$ & 0.071 & -0.071 & \\
\hline $\mathrm{HCN}: \mathrm{PH}_{2} \mathrm{~F}$ & 0.014 & -0.014 & \\
\hline $\mathrm{PH}_{2} \mathrm{~F}: \mathrm{BeH}_{2}(\mathrm{~A})$ & & 0.020 & -0.020 \\
\hline $\mathrm{PH}_{2} \mathrm{~F}: \mathrm{BeH}_{2}(\mathrm{~B})$ & & -0.004 & 0.004 \\
\hline $\mathrm{PH}_{2} \mathrm{~F}: \mathrm{BeH}_{2}(\mathrm{C})$ & & 0.019 & -0.019 \\
\hline $\mathrm{PH}_{2} \mathrm{~F}: \mathrm{BeF}_{2}(\mathrm{~A})$ & & 0.028 & -0.028 \\
\hline $\mathrm{PH}_{2} \mathrm{~F}: \mathrm{BeF}_{2}(\mathrm{~B})$ & & 0.020 & -0.020 \\
\hline $\mathrm{PH}_{2} \mathrm{~F}: \mathrm{BeF}_{2}(\mathrm{C})$ & & 0.025 & -0.025 \\
\hline $\mathrm{PH}_{2} \mathrm{~F}: \mathrm{BeCl}_{2}(\mathrm{~A})$ & & 0.032 & -0.032 \\
\hline $\mathrm{PH}_{2} \mathrm{~F}: \mathrm{BeCl}_{2}(\mathrm{~B})$ & & 0.012 & -0.012 \\
\hline $\mathrm{PH}_{2} \mathrm{~F}: \mathrm{BeCl}_{2}(\mathrm{C})$ & & 0.030 & -0.030 \\
\hline $\mathrm{H} 3 \mathrm{~N}: \mathrm{PH} 2 \mathrm{~F}: \mathrm{BeH} 2(\mathrm{~A})$ & 0.190 & -0.145 & -0.045 \\
\hline H3N:PH2F:BeH2 (B) & 0.184 & -0.156 & -0.028 \\
\hline H3N:PH2F:BeH2 (C) & 0.177 & -0.135 & -0.042 \\
\hline H3N:PH2F:BeF2 (A) & 0.207 & -0.161 & -0.046 \\
\hline H3N:PH2F:BeF2 (B) & 0.201 & -0.162 & -0.039 \\
\hline H3N:PH2F:BeF2 (C) & 0.196 & -0.155 & -0.042 \\
\hline H3N:PH2F:BeCl2 (A) & 0.239 & -0.181 & -0.059 \\
\hline $\mathrm{H} 3 \mathrm{~N}: \mathrm{PH} 2 \mathrm{~F}: \mathrm{BeCl} 2$ (B) & 0.232 & -0.186 & -0.046 \\
\hline $\mathrm{H} 3 \mathrm{~N}: \mathrm{PH} 2 \mathrm{~F}: \mathrm{BeCl} 2(\mathrm{C})$ & 0.228 & -0.175 & -0.053 \\
\hline CH2HN:PH2F:BeH2 (A) & 0.190 & -0.145 & -0.045 \\
\hline CH2HN:PH2F:BeH2 (B) & 0.186 & -0.157 & -0.028 \\
\hline CH2HN:PH2F:BeH2 (C) & 0.181 & -0.137 & -0.044 \\
\hline CH2HN:PH2F:BeF2 (A) & 0.205 & -0.158 & -0.047 \\
\hline CH2HN:PH2F:BeF2 (B) & 0.201 & -0.161 & -0.040 \\
\hline CH2HN:PH2F:BeF2 (C) & 0.198 & -0.155 & -0.043 \\
\hline $\mathrm{CH} 2 \mathrm{HN}: \mathrm{PH} 2 \mathrm{~F}: \mathrm{BeCl} 2(\mathrm{~A})$ & 0.235 & -0.175 & -0.060 \\
\hline $\mathrm{CH} 2 \mathrm{HN}: \mathrm{PH} 2 \mathrm{~F}: \mathrm{BeCl} 2$ (B) & 0.230 & -0.184 & -0.047 \\
\hline $\mathrm{CH} 2 \mathrm{HN}: \mathrm{PH} 2 \mathrm{~F}: \mathrm{BeCl} 2(\mathrm{C})$ & 0.230 & -0.175 & -0.055 \\
\hline
\end{tabular}




\begin{tabular}{|l|l|l|l|}
\hline HCN:PH2F:BeH2 (A) & 0.055 & -0.022 & -0.033 \\
\hline HCN:PH2F:BeH2 (B) & 0.054 & -0.039 & -0.015 \\
\hline HCN:PH2F:BeH2 (C) & 0.050 & -0.017 & -0.033 \\
\hline & & & \\
\hline HCN:PH2F:BeF2 (A) & 0.064 & -0.028 & -0.036 \\
\hline HCN:PH2F:BeF2 (B) & 0.062 & -0.033 & -0.029 \\
\hline HCN:PH2F:BeF2 (C) & 0.060 & -0.027 & -0.033 \\
\hline & & & \\
\hline HCN:PH2F:BeCl2 (A) & 0.083 & -0.037 & -0.046 \\
\hline HCN:PH2F:BeCl2 (B) & 0.080 & -0.050 & -0.030 \\
\hline HCN:PH2F:BeCl2 (C) & 0.078 & -0.037 & -0.041 \\
\hline
\end{tabular}

\begin{tabular}{|l|c|c|c|}
\hline & N-Base & $\mathrm{PH}_{2} \mathrm{~F}$ & BeR \\
\hline $\mathrm{PH}_{2} \mathrm{~F}: \mathrm{BeCO}_{3}$ & -0.059 & 0.059 & \\
\hline $\mathrm{PH}_{2} \mathrm{~F}: \mathrm{BeSO}_{4}$ & -0.064 & 0.064 & \\
\hline & & & \\
\hline $\mathrm{H}_{3} \mathrm{~N}: \mathrm{PH}_{2} \mathrm{~F}: \mathrm{BeCO}_{3}$ & 0.289 & -0.208 & -0.081 \\
\hline $\mathrm{H}_{2} \mathrm{CHN}: \mathrm{PH}_{2} \mathrm{~F}: \mathrm{BeCO}_{3}$ & 0.279 & -0.198 & -0.082 \\
\hline $\mathrm{HCN}: \mathrm{PH}_{2} \mathrm{~F}: \mathrm{BeCO}_{3}$ & 0.130 & -0.060 & -0.070 \\
\hline & & & \\
\hline $\mathrm{H}_{3} \mathrm{~N}: \mathrm{PH}_{2} \mathrm{~F}: \mathrm{BeSO}_{4}$ & 0.298 & -0.212 & -0.087 \\
\hline $\mathrm{H}_{2} \mathrm{CHN}: \mathrm{PH}_{2} \mathrm{~F}: \mathrm{BeSO}_{4}$ & 0.289 & -0.202 & -0.087 \\
\hline $\mathrm{HCN}: \mathrm{PH}_{2} \mathrm{~F}: \mathrm{BeSO}_{4}$ & 0.143 & -0.067 & -0.076 \\
\hline
\end{tabular}

